\title{
Count Rate
}

National Cancer Institute

\section{Source}

National Cancer Institute. Count Rate. NCI Thesaurus. Code C94878.

The number of discrete events, i.e. counts, which occur per unit time. 